13

\title{
Траекторный анализ в коллекторе с многоступенчатой рекуперацией энергии для прототипа гиротрона DEMO. Часть II. Тороидальное магнитное поле
}

\author{
(C) О.И. Лукша, ${ }^{1}$ П.А. Трофиммов, ${ }^{1}$ В.Н. Мануилов, ${ }^{2,3}$ М.Ю. Глявин ${ }^{3}$ \\ ${ }^{1}$ Санкт-Петербургский политехнический университет Петра Великого, \\ 195251 Санкт-Петербург, Россия \\ ${ }^{2}$ Нижегородский государственный университет им. Н.И. Лобачевского, \\ 603950 Нижний Новгород, Россия \\ ${ }^{3}$ Институт прикладной физики РАН, \\ 603950 Нижний Новгород, Россия \\ e-mail: louksha@rphf.spbstu.ru
}

Поступило в Редакцию 11 января 2021 г.

В окончательной редакции 11 января 2021 г.

Принято к публикации 7 февраля 2021 г.

\begin{abstract}
Представлены результаты моделирования коллектора с 4-х ступенчатой рекуперацией остаточной энергии пучка для прототипа гиротрона, разрабатываемого для проекта DEMO. Для пространственной сепарации электронов с разной энергией используется азимутальное магнитное поле, создаваемое тороидальным соленоидом. Повышение эффективности рекуперации и снижение потока электронов, отраженных от коллектора, достигается за счет снижения разброса радиального положения ведущих центров электронных траекторий при оптимальных параметрах тороидального соленоида, а также за счет использования секционированного электронного пучка. Траекторный анализ отработанного потока с распределениями электронов по компонентам скорости и координаты, приближенными к полученным в экспериментах с мощными гиротронами, показал возможность достижения полного КПД гиротрона более $80 \%$, близкого к максимальному КПД при идеальной сепарации фракций электронного пучка с разной энергией.
\end{abstract}

Ключевые слова: СВЧ электроника, гиротрон, электронный поток, рекуперация энергии.

DOI: 10.21883/JTF.2021.07.50960.5-21

\section{Введение}

Использование коллекторов с одноступенчатой рекуперацией позволяет повысить полный КПД мощных гиротронов, предназначенных для нагрева плазмы в установках управляемого термоядерного синтеза (УТС), до $50-55 \%$ [1-4]. Однако для нового поколения установок УТC, таких как проект DEMO, требуются гиротроны с полным КПД, превышающим 60\% [5]. Для достижения столь высокого КПД могут быть использованы системы c многоступенчатой рекуперацией остаточной энергии винтового электронного потока (ВЭП), в которых необходимо обеспечить разделение электронных фракций с разной энергией в пространстве и осаждение этих фракций на секции коллектора под различными тормозящими потенциалами. Показано, что пространственное разделение электронов может быть достигнуто в результате их радиального дрейфа в скрещенных электрическом и магнитном полях [6-8]. В последнее время в СПбПУ были проведены исследования, в которых для такого дрейфа используется азимутальное магнитное поле [9-11]. Была разработана конструкция коллектора с четырехступенчатой рекуперацией для гиротрона СПбПУ с частотой $74.2 \mathrm{GHz}$ и выходной мощностью $\sim 100 \mathrm{~kW}[12-15]$, в которой для формирования азимутального магнитного поля применяется тороидальный соленоид. В результа- те численного моделирования процессов в гиротроне СПбПУ с четырехступенчатой рекуперацией было получено значение полного КПД этого прибора, равное $71.2 \%$ [10].

В первой части данной работы [11] подходы к разработке коллектора с многоступенчатой рекуперацией, опробованные в гиротроне СПбПУ, были применены для прототипа гиротрона, который разрабатывается для проекта DEMO [16,17]. Основные параметры данного гиротрона: частота генерации $f=250 \mathrm{GHz}$, ускоряющее напряжение $U_{0}=55 \mathrm{kV}$, индукция магнитного поля в резонаторе $B_{0}=9.57 \mathrm{~T}$, ток пучка $I_{b}=10 \mathrm{~A}$. В работе [11] для случая четырехступенчатой рекуперации были определены распределения электрического и магнитного полей в коллекторной области этого гиротрона, при которых достигается эффективная сепарация электронов отработанного ВЭП при малом отражении электронов от коллектора в сторону резонатора. Использовалось идеализированное распределение азимутального магнитного поля, создаваемое проводником, расположенным на оси прибора. В режиме работы гиротрона с электронным КПД $36 \%$ в результате четырехступенчатой рекуперации было получено максимальное значение полного КПД, равное $84.3 \%$, при коэффициенте отражения электронов от коллектора $\sim 0.01 \%$. 
В реальном приборе азимутальное магнитное поле может быть создано с помощью соленоида с тороидальной намоткой. В настоящей работе представлены результаты моделирования такого соленоида для прототипа гиротрона DEMO. На основании данных траекторного анализа отработанного ВЭП были выполнены оценки полного КПД гиротрона и рассчитаны распределения плотности мощности пучка, осаждающегося на электродах коллектора. Все расчеты были произведены с помощью программного пакета $3 D$-моделирования CST Studio Suite [18].

\section{1. Модель тороидального соленоида}

При разработке коллектора с рекуперацией для прототипа гиротрона DEMO предполагалось, что его размеры (длина и радиус цилиндрической части) не должны заметно отличаться от соответствующих размеров коллектора без рекуперации [16,17]. Внутренний радиус цилиндрической части коллектора $R_{\text {coll }}$ был выбран равным $160 \mathrm{~mm}$, а расстояние от центра резонатора до задней торцевой плоскости коллектора - $1500 \mathrm{~mm}$. Трехмерное изображение коллекторной области гиротрона показано на рис. 1. Рекуперация осуществляется в цилиндрической части корпуса коллектора 1 , где расположены секции-электроды 5 под тормозящими потенциалами. В этой области корректирующие катушки 2 в комбинации с катушками основной магнитной системы гиротрона создают близкое к однородному продольное магнитное поле. Для создания азимутального магнитного поля используется тороидальный соленоид 3. Такой соленоид позволяет сконцентрировать магнитное поле в локальной области, ограниченной внутренней и наружной обмотками и торцевыми проводниками. Торцевые проводники, расположенные со стороны, ближней к резонатору, препятствуют прохождению электронов в область рекуперации. В данной конструкции соленоида эти проводники собраны в две радиальные связки 4. Это повышает долю электронов, проходящих в область рекуперации. Однако при этом увеличивается амплитуда паразитного магнитного поля в прилегающей к связкам

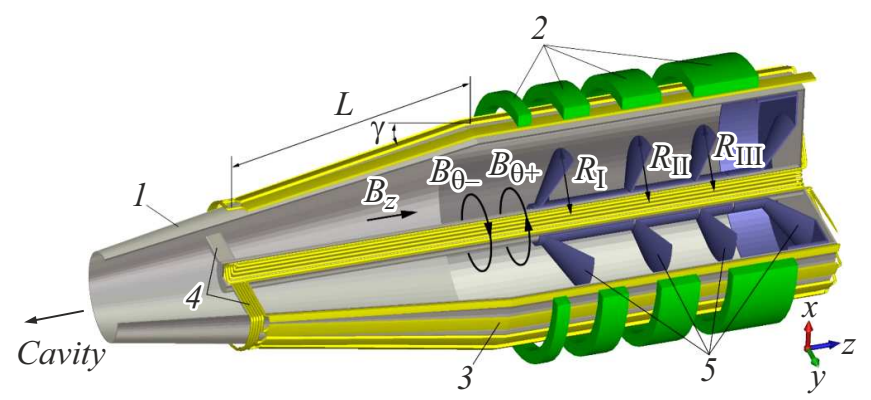

Рис. 1. Схематическое изображение коллекторной области гиротрона: 1 - корпус коллектора, $2-$ корректирующие катушки, 3 - тороидальный соленоид, 4 - связки, 5 - секции коллектора.
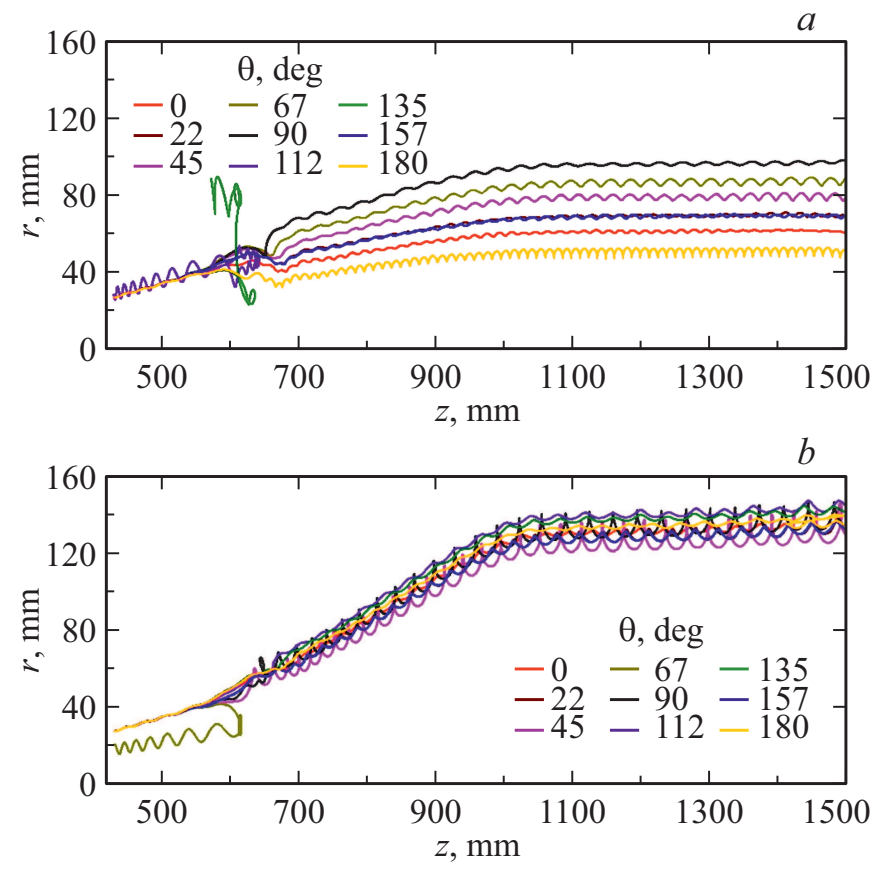

Рис. 2. Проекции траекторий „одиночных“ электронов с различной азимутальной координатой $\theta$ точки старта в диапазоне от $0^{\circ}$ до $180^{\circ}$ при положительном $(a)$ и отрицательном $(b)$ направлениях азимутального магнитного поля. Азимутальное положение связок соответствует координатам $\theta=90^{\circ}$ и $270^{\circ}$, а продольное положение - координате $z=630 \mathrm{~mm}$.

области, которое негативно влияет на проходящие там траектории электронов. Проводники внутренней обмотки и связок тороидального соленоида расположены в трубках (рис. 1), которые отделяют вакуумное рабочее пространство коллектора от этого соленоида.

Для снижения влияния паразитного магнитного поля связок была выполнена оптимизация параметров тороидального соленоида. Для этого использовались результаты расчетов траекторий „одиночных“ электронов. Задавались точечные источники частиц в центральной плоскости резонатора $(z=0)$. Начальная энергия и радиальная координата у всех частиц были одинаковыми и равнялись соответственно $36 \mathrm{kV}$ и $3.85 \mathrm{~mm}$, а азимутальная координата $\theta$ была различной. В области рекуперации $(z>1000 \mathrm{~mm})$ индукция азимутального магнитного поля $B_{\theta}$ была равна примерно $0.08 \mathrm{~T}$, а продольного поля $B_{z}$ - примерно 0.016 Т. Эти значения были определены на радиусе, равном радиусу электронной траектории в отсутствии поля тороидального соленоида.

На рис. 2 показаны проекции электронных траекторий на плоскость $r-z$ для разных значений начальной азимутальной координаты $\theta$, рассчитанные при положительном $B_{\theta+}$ (рис. $\left.2, a\right)$ и отрицательном $B_{\theta-}$ (рис. $\left.2, b\right)$ направлениях азимутального магнитного поля (рис. 1). Видно, что при положительном направлении этого поля имеется заметный разброс радиальных координат траекторий для электронов с разными $\theta$. Причем этот 
разброс в гиротроне DEMO больше, чем в гиротроне СПбПУ [10] при близких параметрах тороидального соленоида. Это связано, в первую очередь, с меньшим значением индукции продольного магнитного поля в коллекторной области гиротрона DEMO. Варьирование длины конусной части соленоида $L$ и угла наклона $\gamma$ (рис. 1) не приводит к заметному снижению радиального разброса электронных траекторий при положительном направлении поля $B_{\theta+}$. Наличие такого разброса приводит к снижению эффективности работы рекуператора. Это связано с тем, что электроны, двигающиеся в тормозящем поле на малых радиусах, после изменения направления продольной скорости могут не попасть на секцию, потенциал которой соответствует энергии этих электронов, а осесть на секциях под более положительным потенциалом. Такие электроны, отразившись, могут также выйти из коллекторной области в сторону резонатора.

Анализ сил, действующих на электроны в области связок, показал, что положительного результата можно добиться, если изменить направление азимутального магнитного поля. В этом случае меняется направление радиальной силы Лоренца, которая действует на электроны, двигающиеся в продольном направлении, при влете в область с азимутальным магнитным полем. Эта сила будет смещать электроны в сторону бо́льших радиусов при отрицательном направлении поля $B_{\theta-}$. Кроме этого, радиальное смещение электронов происходит в результате действия силы, связанной с изменением продольного магнитного поля вдоль координаты $z$. При отрицательном направлении поля $B_{\theta-}$ одна из указанных сил частично компенсирует другую. Это приводит к уменьшению разброса радиальных координат электронных траекторий при смене направления азимутального поля. Дополнительного снижения этого разброса можно добиться за счет оптимизации параметров тороидального соленоида $L$ и $\gamma$. На рис. $2, b$ показаны проекции электронных траекторий, рассчитанные при отрицательном направлении поля $B_{\theta-}$ и оптимальных значениях $L=350 \mathrm{~mm}$ и $\gamma=9.5^{\circ}$. Видно, что радиальный разброс траекторий в этом случае заметно меньше, чем при положительном направлении поля $B_{\theta+}$.

Смена направления азимутального поля неизбежно приводит к необходимости изменения геометрии секций коллектора по сравнению с описанной в первой части работы [11] для идеализированного распределения магнитного поля. При отрицательном направлении дрейф электронов в скрещенных $E_{z}$ и $B_{\theta}$ полях будет направлен в сторону меньших радиусов. Секции имеют конусную форму (рис. 1), при которой амплитуда продольной компоненты электрического поля $E_{z}$ изменяется незначительно вдоль координаты $z$ в области рекуперации. У каждой из секций имеются цилиндрические основания, которые служат для экранирования рабочего пространства, где движутся электроны, от заземленных частей корпуса коллектора. Радиусы краев первых трех секций $R_{\mathrm{I}}, R_{\mathrm{II}}$ и $R_{\mathrm{III}}$ следует выбрать таким образом, чтобы в отсутствие напряжения на секциях электроны не осаждались на их поверхности. Для этого могут быть использованы результаты расчетов траекторий „одиночных“ электронов (рис. 2). На начальном этапе моделирования эти радиусы были заданы равными $105 \mathrm{~mm}$.

\section{2. Результаты траекторного анализа в коллекторе с тороидальным соленоидом}

При выполнении траекторного анализа источник электронов находился в центральной плоскости резонатора $(z=0)$. В случае однородного пучка в этой плоскости располагались 936 центров эмиссии, каждый из которых являлся источником 76 частиц с различными значениями начальной энергии, тока, питч-фактора и фазы циклотронного вращения. Энергии частиц задавались по типичному энергетическому спектру отработанного ВЭП в мощных гиротронах, соответствующему режиму с электронным КПД $\eta_{\mathrm{el}}=36 \%$ [11].

Оптимизация параметров тороидального соленоида позволила уменьшить влияние магнитного поля связок. Однако после такой оптимизации еще остаются электроны, которые движутся в непосредственной близости от связок в области, где продольное магнитное поле может изменить свое направление [19]. Реверс продольного магнитного поля проводит к заметному искривлению траекторий электронов и, как следствие, к их возможному отражению от коллектора в сторону резонатора (см. траекторию с $\theta=67^{\circ}$ на рис. $2, b$ ). Как и ранее при моделировании в гиротроне СПбПУ [10], в гиротроне DEMO было выполнено секционирование ВЭП, что позволило практически исключить отражение электронов от коллектора. В секционированном ВЭП отсутствуют электроны в двух (по числу связок тороидального соленоида) азимутальных секторах. Для создания такого ВЭП в реальном приборе может быть использован секционированный катод, у которого с двух азимутальных секторов термопояска удалено эмитирующее покрытие. Очевидно, что в результате секционирования снижается качество пучка, а также эффективность его взаимодействия с высокочастотным полем в резонаторе. Однако, как показали расчеты в гиротроне СПбПУ, это снижение невелико. Выходная СВЧ мощность этого гиротрона уменьшалась с 141 до $138 \mathrm{~kW}$ при переходе от однородного к секционированному катоду, у которого отсутствовала эмиссия с двух секторов протяженностью $70^{\circ}$ в азимутальном направлении [10].

При выполнении траекторного анализа в коллекторной области гиротрона DEMO исследовалась возможность достижения максимальной эффективности рекуперации, т.е. снижения до минимума величины мощности $P_{\text {diss}}$, рассеиваемой в виде тепла при осаждении электронов на стенках коллектора. Подобно расчетам с идеализированным распределением магнитного поля [11], 


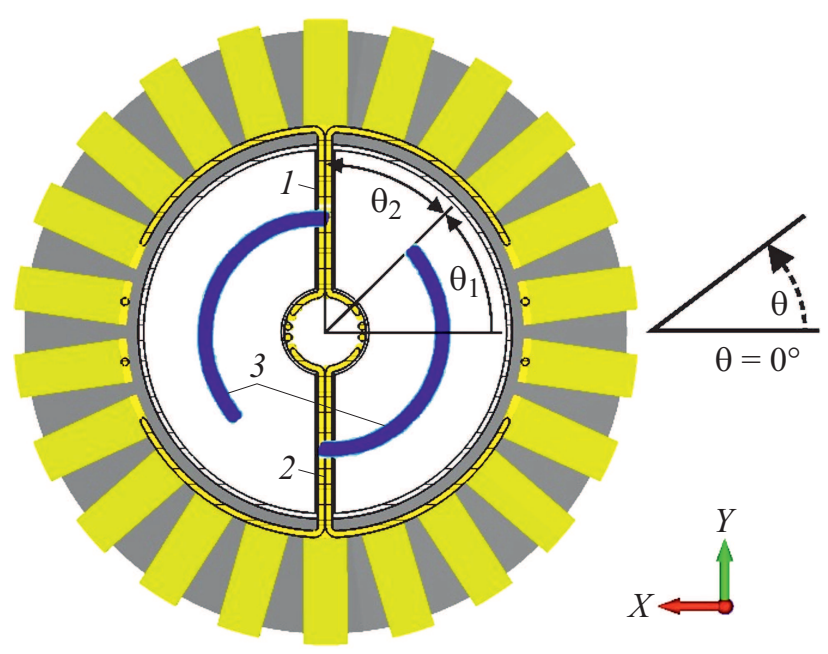

Рис. 3. Изображение элементов тороидального соленоида $(1,2-$ связки) и секционированного электронного пучка 3 в плоскости поперечного сечения $x-y$ при наблюдении со стороны резонатора. Секторы ВЭП, в которых отсутствуют электроны, смещены друг относительно друга на $180^{\circ}$. Углы $\theta_{1}$ и $\theta_{2}$ показаны только для одного из секторов.

в области рекуперации значения индукций азимутальной $B_{\theta}$ и продольной $B_{z}$ компонент магнитного поля были равны соответственно 0.08 и $0.016 \mathrm{~T}$, а значения напряжений на секциях $U_{\mathrm{I}}=-23.6 \mathrm{kV}, U_{\mathrm{II}}=-29.1 \mathrm{kV}$, $U_{\mathrm{III}}=-32.4 \mathrm{kV}, U_{\mathrm{IV}}=-37.8 \mathrm{kV}$ при заземленном корпусе коллектора. Для достижения минимальной мощности $P_{\text {diss }}$ регулировался радиус основания первой секции $R_{\mathrm{I}}$, а также углы $\theta_{1}$ и $\theta_{2}$ (рис. 3 ), которые характеризуют азимутальное положение секторов ВЭП, в которых отсутствуют электроны, по отношению к связкам тороидального соленоида и протяженность (длину) этих секторов. По рассчитанной мощности $P_{\text {diss }}$ определялся полный КПД гиротрона $\eta_{t}$, используя соотношение

$$
\eta_{t}=\frac{P_{\mathrm{RF}}}{P_{\mathrm{RF}}+P_{\mathrm{diss}}}
$$

где $P_{\mathrm{RF}}=\eta_{e l} I_{b} U_{0}=198 \mathrm{~kW}$ - выходная СВЧ мощность.

На рис. 4 показаны зависимости полного КПД $\eta_{t}$ и коэффициента отражения $K_{\text {ref }}=I_{\text {ref }} / I_{b}$, где $I_{\text {ref }}-$ ток электронов, отраженных от коллектора и проходящих через входную плоскость в сторону резонатора, от параметров $\theta_{1}, \theta_{2}$ и $R_{\mathrm{I}}$. При изменении длины секторов $\theta_{2}$ полный ток пучка поддерживался равным 10 А. Судя по приведенным зависимостям, оптимальным является положение секторов, при котором они находятся в диапазонах углов примерно от $45^{\circ}$ до $90^{\circ}$ и от $225^{\circ}$ до $270^{\circ}$ (рис. 3). В этих областях суммарное продольное магнитное поле, образованное основной магнитной системой гиротрона, корректирующими катушками, а также связками проводов тороидального соленоида, меняет свое направление на обратное. С увеличением длины секторов $\theta_{2}$ эффективность работы рекуператора, очевидно, возрастает - повышается полный КПД и снижается коэффициент отражения. Следует при этом отметить, что для определения более точной зависимости $\eta_{t}\left(\theta_{2}\right)$ необходимо учесть отмеченное выше снижение электронного КПД $\eta_{e l}$ при увеличении угла $\theta_{2}$. С практической точки зрения важно также иметь в виду, что для формирования секционированного ВЭП потребуется соответствующий секционированный катод с повышенной плотностью тока эмиссии и температурой, которые, в свою очередь, увеличиваются с ростом $\theta_{2}$. Для дальнейших расчетов были выбраны следующие оптимальные значения углов $\theta_{1}=45^{\circ}$ и $\theta_{2}=45^{\circ}$. Отметим, что найденные решения по оптимизации параметров тороидального соленоида в гиротроне DEMO позволили уменьшить длину секторных вырезов ВЭП в этом гиротроне по сравнению с гиротроном СПбПУ [10].

Увеличение радиуса первой секции приводит к снижению полного КПД (рис. 4,c) из-за того, что увеличивается доля электронов с большими энергиями, перехватываемых этой секцией и не проходящих в область более отрицательных потенциалов. При малых значениях радиуса $R_{\mathrm{I}}$ дрейфового расстояния, которое проходят отразившиеся электроны, становится недоста-
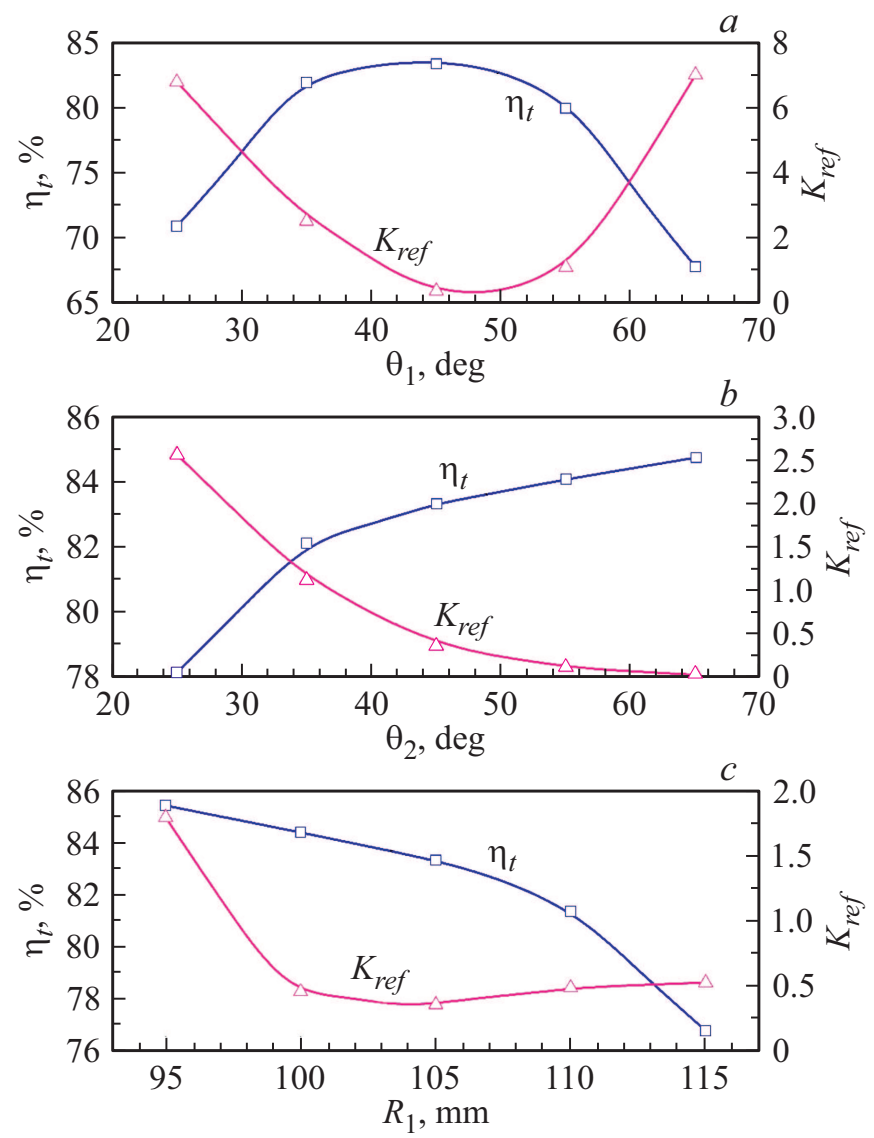

Рис. 4. Зависимости полного КПД гиротрона $\eta_{t}$ и коэффициента отражения электронов от коллектора $K_{\text {ref }}$ от угла $\theta_{1}$ при $\theta_{2}=45^{\circ}$ и $R_{I}=105 \mathrm{~mm}(a)$, от угла $\theta_{2}$ при $\theta_{1}=45^{\circ}$ и $R_{I}=105 \mathrm{~mm}(b)$ и от радиуса первой секции $R_{I}$ при $\theta_{1}=45^{\circ}$ и $\theta_{2}=45^{\circ}(c)$. 
точно для перехвата этих электронов первой секцией. Такие электроны выходят из коллекторной области, что приводит к повышению $K_{\text {ref. Результаты, описанные }}$ ниже, были получены при $R_{\mathrm{I}}=105 \mathrm{~mm}$.

Распределение мощности ВЭП по электродам коллектора характеризуют данные, приведенные в таблице. Здесь указаны значения мощности, рассеиваемой на секциях $P_{\mathrm{I}}-P_{\mathrm{IV}}$ и на корпусе коллектора $P_{\text {coll }}$, сумма этих значений $P_{\text {diss }}$, коэффициент отражения $K_{\text {ref }}$ и полный КПД $\eta_{t}$. На рис. 5 показано положение частиц в плоскости $x=0$, полученное как результат пересечения винтообразных электронных траекторий с этой плоскостью. Видно, что по мере движение электронов в тормозящем электрическом поле происходит уменьшение их энергии и смещение в радиальном направлении, в данном случае в сторону меньших радиусов, с последующим осаждением на секциях. С ростом начальной энергии электронов они проходят бо́льшее расстояние вдоль оси $z$ и осаждаются на секции под более отрицательным потенциалом.

Важный результат проведенных исследований состоит в том, что в гиротроне с тороидальным соленоидом были достигнуты значения полного КПД и коэффициента отражения от коллектора, незначительно отличающиеся от подобных значений для идеализированного распределения азимутального магнитного поля [11]. Это является следствием эффективного подавления паразитного влияния магнитного поля связок в результате изменения направления поля $B_{\theta}$, а также выбора оптимальных значений параметров $L$ и $\gamma$. При использовании тороидального соленоида часть электронов оседает на заземленном корпусе коллектора (см. таблицу), поскольку при измененном направлении азимутального магнитного поля радиальная сила Лоренца смещает электроны на бо́льшие радиусы. Однако рассеиваемая на корпусе коллектора мощность не велика по сравнению с мощностью на секциях, что не приводит к заметному снижению полного КПД.

Как было отмечено при расчете рекуператора с идеализированным магнитным полем [11], снижения полного КПД следует ожидать, если в расчетах вместо ВЭП с

Результаты траекторного анализа в коллекторе с 4-х струпенчатой рекуперацией для прототипа гиротрона DEMO $\left(\theta_{1}=45^{\circ}, \theta_{2}=45^{\circ}, R_{\mathrm{I}}=105 \mathrm{~mm}\right)$

\begin{tabular}{l|c}
\hline Параметр & Значение \\
\hline$P_{\mathrm{I}}, \mathrm{kW}$ & 9.03 \\
$P_{\mathrm{II}}, \mathrm{kW}$ & 5.61 \\
$P_{\mathrm{III}}, \mathrm{kW}$ & 11.50 \\
$P_{\mathrm{IV}}, \mathrm{kW}$ & 12.84 \\
$P_{\text {coll }}, \mathrm{kW}$ & 0.42 \\
$P_{\text {diss }}, \mathrm{kW}$ & 39.4 \\
$K_{\mathrm{ref}}, \%$ & 0.35 \\
$\eta_{t}, \%$ & 83.4
\end{tabular}

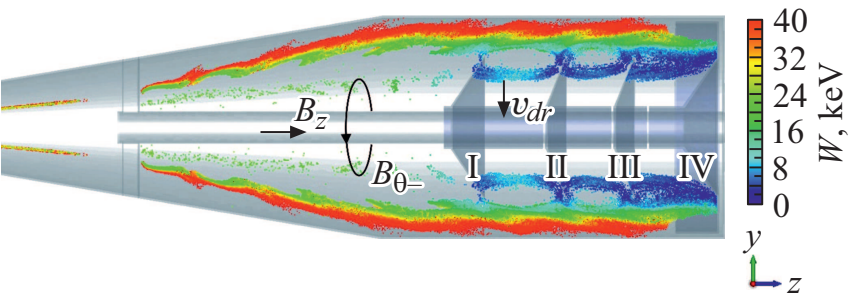

Рис. 5. Положение частиц в плоскости $x=0$ (цвет соответствует энергии частиц $W$ ). Показаны секции коллектора I-IV, а также направления продольного $B_{z}$ и азимутального $B_{\theta}$ магнитных полей и скорости дрейфа электронов $v_{d r}$.

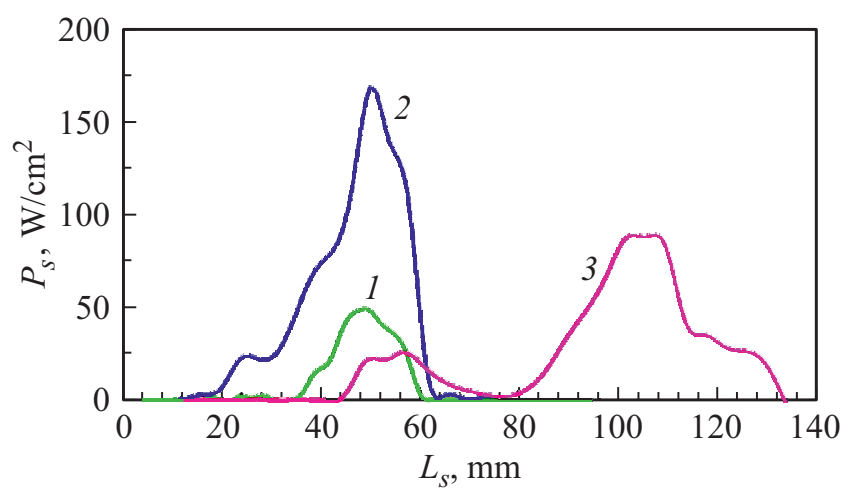

Рис. 6. Зависимости усредненной плотности мощности $P_{s}$ осаждающегося ВЭП от расстояния $L_{s}$ вдоль образующей секций III и IV коллектора. Показаны зависимости для передней (1) и задней (2) поверхностей секции III и для передней (3) поверхности секции IV.

упрощенным энергетическим спектром будет использоваться отработанный электронный поток, полученный в результате численного моделирования процессов в резонаторе данного гиротрона. В рамках такого моделирования может быть использован первичный ВЭП, сформированный в электронно-оптической системе (ЭОС) с секционированным эмиттером. Подобные расчеты были выполнены для гиротрона СПбПУ [10]. Если принять во внимание результаты этих расчетов, то можно предположить, что и в прототипе гиротрона DEMO может быть обеспечена высокая эффективность рекуперации и высокий полный КПД в случае использования отработанного пучка с распределением частиц по скоростям и координатам, в бо́льшей степени приближенным к реальным условиям.

Распределение тепловой нагрузки по поверхности секций коллектора характеризуют зависимости на рис. 6 и 7. Эти зависимости приведены для секций III и IV, на которых рассеивается наибольшая мощность. Для получения значений плотности мощности $P_{s}$ было выполнено усреднение, при котором площадь осаждения каждой из электронных траекторий (трубок тока) принималась приблизительно равной квадрату расстояния между соседними траекториями. На рис. 6 показана величина $P_{s}$, усредненная по азимутальной координате, 

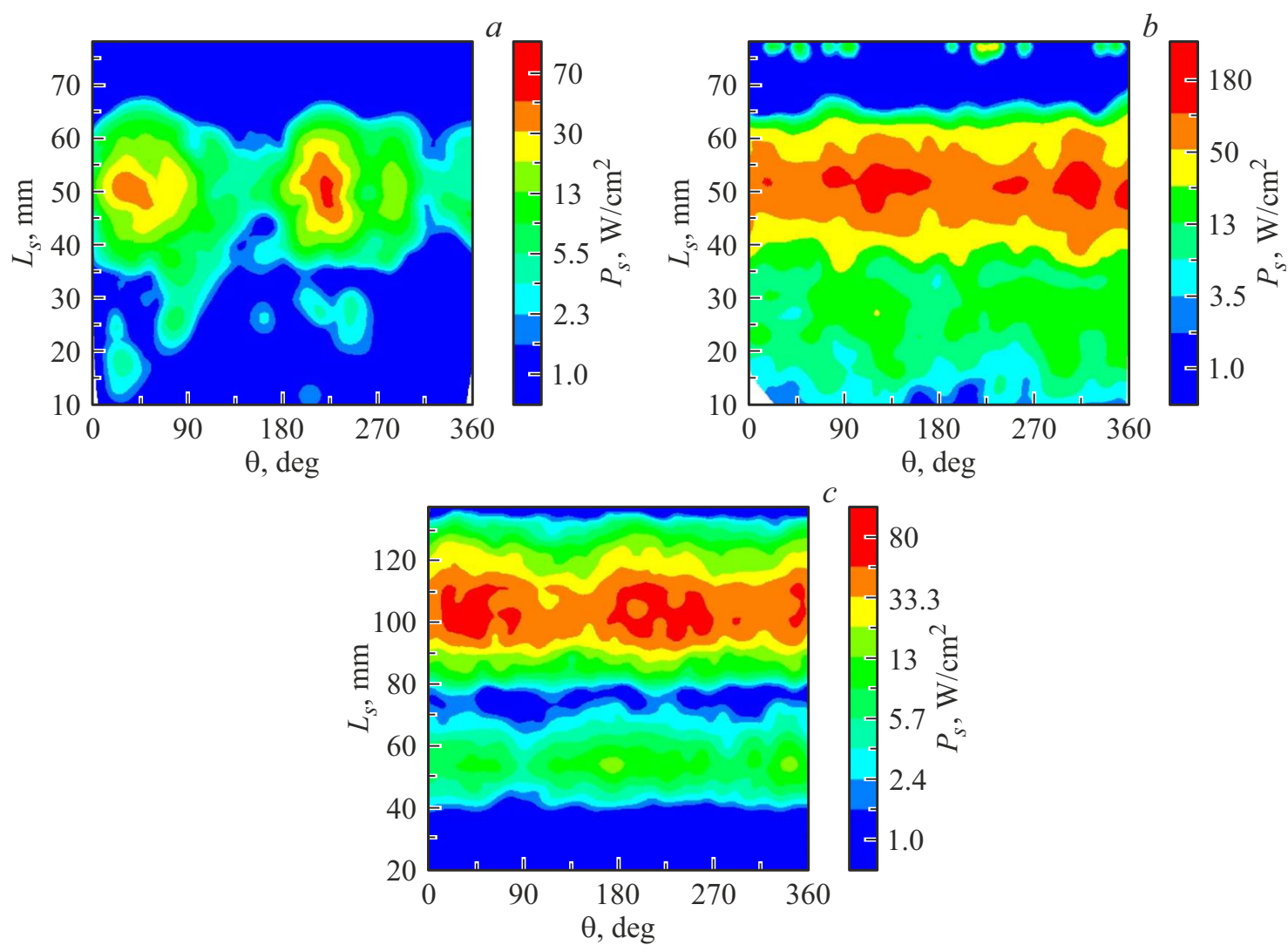

Рис. 7. Распределения плотности мощности $P_{s}$ осаждающегося ВЭП по передней $(a)$ и задней $(b)$ поверхностям секции III и передней $(c)$ поверхности секции IV.

в зависимости от расстояния $L_{s}$ вдоль образующей конусных секций. Для секции III зависимости $P_{s}\left(L_{s}\right)$ приведены отдельно для передней (ближней к резонатору) и задней поверхностей. Значение $L_{s}=0$ соответствует переходу конусных частей секций к их цилиндрическому основанию (рис. 1). Край секции III (при $r=R_{\text {III }}$ ) при этом соответствует $L_{s}=78.8 \mathrm{~mm}$. Двумерные распределения плотности мощности по поверхности секций приведены на рис. 7. В отличие от идеализированного распределения магнитного поля [11] в коллекторе с тороидальным соленоидом распределение плотности мощности осаждающегося ВЭП неоднородно вдоль азимутальной координаты, что связано с использованием секционированного ВЭП и влиянием магнитного поля связок. Однородность распределения $P_{s}$ вдоль $L_{s}$ и $\theta$, очевидно, будет больше, если в расчетах использовать отработанный ВЭП с большим числом частиц, начальные координаты и скорости которых будут различаться.

Приведенные распределения плотности мощности показывают, что в разработанной конструкции рекуператора происходит значительное радиальное смещение частиц, что способствует их осаждению примерно в центральной области конусных поверхностей секций (см., например, рис. $7, b)$. В результате дальнейшей оптимизации геометрии секций, например, при регулировании радиуса основания и угла наклона конуса, можно добить- ся снижения плотности мощности осаждающегося ВЭП. Это может позволить обеспечить приемлемую тепловую нагрузку на коллектор при переходе к мегаваттным уровням мощности в гиротроне для проекта DEMO. Хотя такой переход, безусловно, потребует дополнительной конструкторской проработки коллектора гиротрона с учетом интенсивного водяного охлаждения.

\section{Заключение}

Таким образом, выполненное в работе моделирование коллектора для гиротрона, который был разработан как прототип для гиротрона DEMO, подтвердило возможности эффективной сепарации электронов отработанного ВЭП, основанной на их дрейфе в скрещенных азимутальном магнитном и аксиальном электрическом полях. Основой этого моделирования послужили данные траекторного анализа, который был выполнен в первой части работы для идеализированного распределения азимутального магнитного поля, создаваемого расположенным на оси прибора проводником [11]. В разработанной конструкции 4-х ступенчатого рекуператора с тороидальным соленоидом, параметры которого были оптимизированы для повышения эффективности рекуперации и снижения отражения электронов от коллектора, был достигнут полный КПД гиротрона, превышающий 
83\%. Дальнейшее развитие этих исследований может быть связано с определением возможностей снижения средней тепловой нагрузки на коллектор и повышения однородности его нагрева при переходе к мегаваттному уровню выходной мощности.

\section{Финансирование работы}

Исследование выполнено за счет гранта Российского научного фонда (проект № 16-12-10010). Часть результатов была получена с использованием вычислительных ресурсов суперкомпьютерного центра Санкт-Петербургского политехнического университета Петра Великого (http://www.scc.spbstu.ru). Разработка прототипа гиротрона для DEMO ведется в рамках проекта Российского научного фонда 19-79-30071 и все требования к ЭОС сформулированы в рамках этого гранта.

\section{Конфликт интересов}

Авторы заявляют, что у них нет конфликта интересов.

\section{Список литературы}

[1] K. Sakamoto, M. Tsuneoka, A. Kasugai, T. Imai, T. Kariya, K. Hayashi, Y. Mitsunaka. Phys. Rev. Lett., 73 (26), 3532 (1994).

[2] M.Y. Glyavin, A.N. Kuftin, N.P. Venediktov, V.E. Zapevalov. Int. J. Infrared Millim., 18, 2129 (1997).

[3] M. Thumm. J. Infrared Millim. Te., 41 (1), 1 (2020).

[4] V.N. Manuilov, M.V. Morozkin, O.I. Luksha, M.Y. Glyavin. Infrared Phys. Techn., 91, 46 (2018).

[5] J. Jelonnek et al. Fusion Eng. Des., 123, 241(2017).

[6] I.Gr. Pagonakis, J.-P. Hogge, S. Alberti, K.A. Avramides, J.L. Vomvoridis. IEEE Tr. Plasma Sci., 36 (2), 469 (2008).

[7] О.И. Лукша, П.А. Трофимов. Письма в ЖТФ, 41 (18), 38 (2015). [O.I. Louksha, P.A. Trofimov. Tech. Phys. Lett., 41 (9), 884 (2015).]

[8] C. Wu, I.G. Pagonakis, K.A. Avramidis, G. Gantenbein, S. Illy, M. Thumm, J. Jelonnek. Phys. Plasmas, 25 (3), 033108 (2018).

[9] O.I. Louksha, P.A. Trofimov. Proc. 18th Int. Vacuum Electronics Conf., IVEC 2017 (London, United Kingdom, 2017), p. 1.

[10] О.И. Лукша, П.А. Трофимов. ЖТФ, 89 (12), 1988 (2019). [O.I. Louksha, P.A. Trofimov. Tech. Phys., 64 (12), 1889 (2019).]

[11] О.И. Лукша, П.А. Трофимов. ЖТФ, 91 (1), 125 (2021). [O.I. Louksha, P.A. Trofimov. Tech. Phys., 66 (1), 118 (2021).]

[12] Д.В. Касьяненко, О.И. Лукша, Б. Пиосчик, Г.Г. Соминский, М. Тумм. Изв. вузов. Радиофизика, 47 (5-6), 463 (2004). [D.V. Kas'yanenko, O.L. Louksha, B. Piosczyk, G.G. Sominsky, M. Thumm. Radiophys. Quant. Electron., 47 (5-6), 414 (2004).

[13] O. Louksha, B. Piosczyk, G. Sominski, M. Thumm, D. Samsonov. IEEE Tr. Plasma Sci., 34 (3), 502 (2006).

[14] Д.В. Борзенков, О.И. Лукша. ЖТФ, 67 (9), 98 (1997). [D.V. Borzenkov, O.I. Luksha. Tech. Phys. 42 (9), 1071 (1997).]
[15] О.И. Лукша, Д.Б. Самсонов, Г.Г. Соминский, А.А. Цапов. ЖТФ, 82 (6), 101 (2012). [O.I. Louksha, D.B. Samsonov, G.G. Sominskii, A.A. Tsapov. Tech. Phys., 57 (6), 835 (2012).]

[16] M. Glyavin, V. Manuilov, M. Morozkin. Proc. 43rd Int. Conf. Infrared, Millimeter, and Terahertz Waves (Nagoya, Japan, 2018), 8510139.

[17] G.G. Denisov et al. Rev. Sci. Instrum., 89 (8), 084702 (2018).

[18] CST Studio Suite. Electromagnetic field simulation software [Электронный ресурс] Режим доступа: https://www.3ds.com/products-services/simulia/products/cststudio-suite/ 